\title{
QUAND LA FIBRE OPTIQUE REND les bâtiments communicants
}

\author{
Robin COHEN-SELMON \\ Alice BASTICK-RUIZ \\ OSMOS Group SA, \\ 37 rue la Pérouse, 75116 Paris \\ jouberteix@osmos-group.com
}

\section{Une promesse: évaluer la santé des stiructuires}

Le système des cordes optiques permet d'évaluer en continu et en temps réel la santé des structures (patrimoine historique, écoles, ponts, tunnels, murs de soutènement, immeubles, etc.). Il est utilisé en France sur la Tour Eiffel, le toit du Stade de France, la Basilique de Vézelay, le Palais Bourbon, mais aussi le Pont Champlain au Canada et la Tour Taipei à Taiwan.

Les secteurs d'applications s'avèrent très nombreux. Quant aux contextes d'intervention, nous en dénombrons quatre. Les trois principaux sont les situations de travaux, de levée de doutes et de gestion de crise (post effondrements, séismes, inondations, incendies, etc.); le quatrième contexte que la société cherche à développer dans les années à venir est la maintenance préventive.

En résumé, cette technologie basée sur des capteurs à fibre optique permet de gérer durablement des ouvrages, d'optimiser les budgets de maintenance à long terme et, par conséquent, de dégager de la marge budgétaire: en offrant une vision

Tous les bâtiments (immeubles, ouvrages d'art, etc.) sont exposés à de nombreux facteurs de défaillances structurelles: vieillissement, affaiblissement lié à une absence de mise aux normes ou à un défaut d'entretien, travaux de rénovation ou travaux mitoyens. Une solution actuellement en plein essor repose sur les capteurs à fibres optiques: cet article présente le système baptisé "cordes optiques", inventé et développé par la société OSMOS, et utilisant des fibres optiques tressées, qui permet de mesurer en temps réel les déformations d'une structure, d'anticiper les opérations de préservation pour assurer leur pérennité et la sécurité des populations.

claire de l'état des ouvrages, leurs clients peuvent en effet effectuer les bons travaux au bon moment, évitant ainsi des dépenses excessives et parfois inutiles.

Le système des cordes optiques est basé sur des capteurs de haute précision qui mesurent les déformations entre deux points avec une résolution micrométrique. Le principal avantage de ces capteurs est qu'ils fonctionnent sans temps mort et qu'ils sont synchronisés. Les évènements «dynamiques» peuvent ainsi être suivis avec une fréquence de mesure pouvant aller jusqu'à $100 \mathrm{~Hz}$, ce qui permet de réaliser un enregistrement continu et de détecter des phénomènes dynamiques tels que des passages de véhicules sur des ponts, des séismes, des chocs, etc. Si aucun évènement dynamique ne survient, un point de mesure est créé toutes les 10 à 60 minutes. On obtient ainsi une mesure dite statique, représentative du comportement de l'ouvrage sur le long terme.

Ce système peut être utilisé en complément d'autres capteurs (extensomètres, inclinomètres, etc.), dont les données sont collectées en temps réel (à distance, via internet; ou directement sur site), pour un traitement complet des informations sur la structure surveillée. Chaque capteur intègre également une sonde de température. Il est ainsi possible de corréler les déformations structurelles avec ce paramètre et donc de distinguer les déformations naturelles de la structure de celles liées aux sollicitations extérieures. La restitution des données enregistrées par la corde optique LIRIS se fait sous la forme de rapports synthétiques reprenant les déformations précises des ouvrages instrumentés et la corrélation avec les variations thermiques.

\section{Modulation \\ d'iontensité lumineuse, comment ça marcche?}

Le procédé consiste à utiliser des fibres optiques tressées pour mesurer en temps réel les déformations d'une structure. Les déformations structurelles entrainent une variation de l'intensité lumineuse du signal de sortie. La quantité de lumière dissipée dans les microcourbures de la tresse est fonction des déformations longitudinales appliquées sur la tresse, proportionnellement aux déformations de la structure. 
L'analyse de la variation de l'intensité d'un signal lumineux, lorsqu'il parcourt la corde optique permet de connaitre l'élongation (ou le raccourcissement) de cette dernière, avec une résolution de $2 \mu \mathrm{m}$ pour une longueur standard de 2 mètres. Cette mesure en base longue permet de s'affranchir des effets locaux propres aux structures inhomogènes (maçonnerie, béton fissuré) afin d'obtenir une information pertinente à l'échelle de la structure entière.

\section{Focus: la surveillance d'un pont}

Voici le cas d'un pont en béton armé situé dans le sud-est de la France, d'une longueur totale de 38 mètres, construit en 1940. Une inspection détaillée datant de 2011 a révélé de nombreuses fissures dans le tablier en béton armé, déjà relevées lors d'une précédente inspection datant de 1987. La collectivité responsable de ce pont a décidé de surveiller plus étroitement l'évolution de cette fissuration. Les fissures les plus significatives sont observées sur les poutres longitudinales, principales structures porteuses de l'ouvrage. Elles constituent un risque certain compte tenu de leur ouverture importante. Dans cette situation, l'objet du suivi structurel est de qualifier très précisément les incidences de la fissuration sur le comportement mécanique général de l'ouvrage, et de surveiller précocement l'apparition éventuelle de comportements anormaux. En plus d'une mesure précise de l'évolution de l'ouverture des fissures les plus importantes, le suivi structurel consiste à mesurer les déformations des poutres afin de qualifier leur flexion et son évolution dans le temps, à court terme sous les sollicitations dynamiques dues aux convois, et à long terme sous l'effet du fluage.

Chaque poutre surveillée est ainsi instrumentée en cordes optiques placées en partie centrale, qui permettent une mesure des déformations avec une résolution de $1 \mu \mathrm{m} / \mathrm{m}$ en base longue ( 2 mètres) plus pertinente pour qualifier la flexion des poutres de 23 mètres qu'une mesure locale.

Après six mois d'instrumentation, l'étude a permis d'analyser l'effet des variations saisonnières de température ainsi que celui des véhicules lourds (environ 50 convois par semaine ont un tonnage estimé à plus de 20 tonnes; les effets de ces convois sont visibles à la fois à mi-travée où les plus lourds génèrent une déformation de $0,175 \%$, et sur les diagonales d'about où ils génèrent une déformation de $0,043 \%$; ces ordres de grandeur correspondent à des déformations acceptables pour une structure en béton armé sous l'effet de charges d'exploitation). Enfin, le retour à l'état initial du tablier est constaté après l'ensemble des passages, et les effets de ces derniers sont stables au cours du temps.

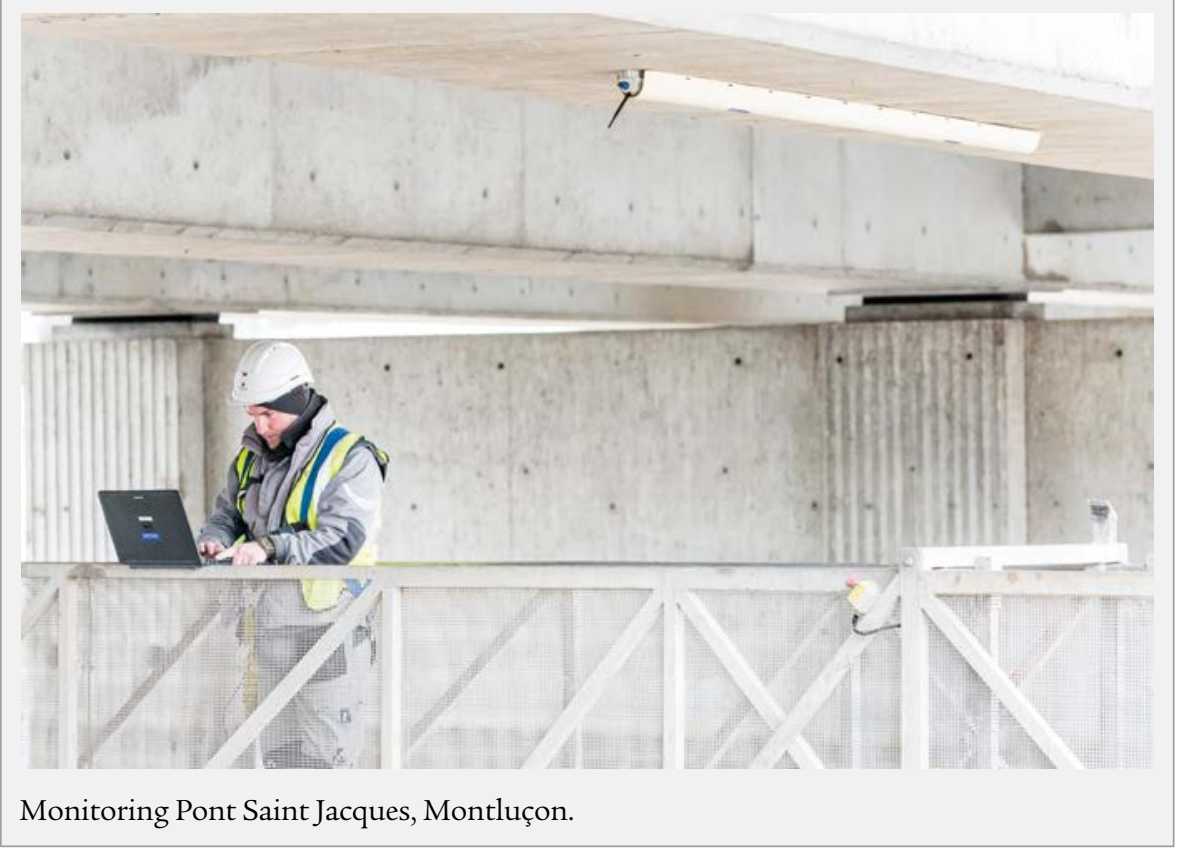

\section{L’analyse de données, un outil utile à la prévention}

L'analyse de données à la fois statiques et dynamiques permet de déterminer l'état de santé actuel de la structure mais aussi de fixer des indices d'évolution de son comportement. Leur progression est évaluée dans le temps afin de donner une représentation précise et fiable du comportement de l'ouvrage et d'anticiper tout besoin d'intervention. De même, la data analysis est source de valeur puisqu'il constitue une base de données en perpétuelle évolution, enrichie avec le temps et permettant d'améliorer la qualité des expertises réalisées.

\section{Nombreux secteurs d'applications}

Ce système trouve naturellement son utilité pour de nombreuses infrastructures (ouvrages d'art, immeubles, patrimoine historique, centres commerciaux, tunnels, murs de soutènement, bâtiments industriels, stades, etc.); chaque type de structure présentant des caractéristiques structurelles qui lui sont propres. Les conditions d'exploitation, le trafic et l'environnement sont à l'origine de nombreux dommages qui imposent aux gestionnaires de ponts une attention accrue. Tassement d'appui, problèmes d'affouillement, fissuration des tabliers en béton, vieillissement de la précontrainte, fluage, fatigue des ponts métalliques... des dommages dont l'origine et l'impact réel sur le comportement mécanique de la structure sont difficilement identifiables via les méthodes classiques.

Autre exemple avec les immeubles de grande hauteur, des structures complexes soumises à des contraintes spécifiques parmi lesquelles une sensibilité particulière aux aléas climatiques et aux tassements différentiels. Dans le cas d'immeubles de grande hauteur, les défaillances structurelles peuvent 
Imegine optit

Wavefront sensors and

adaptive optics for

optical metrology,

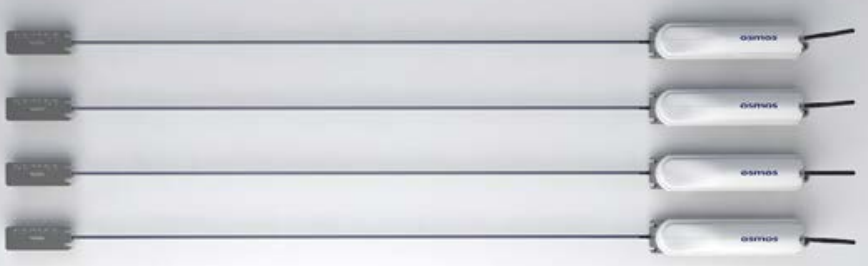

Figure 1.

Corde optique LIRIS.

avoir de sérieuses conséquences pour la sécurité des usagers et impliquer d'importants coûts de renforcement et de maintenance.

Enfin, les monuments historiques présentent des intérêts architecturaux et artistiques qu'il convient de préserver. Ces monuments font l'objet de précautions et de dispositions nécessaires mais contraignantes pour assurer leur conservation, notamment en ce qui concerne les interventions d'entretien, de réparation ou de restauration.

Avec leurs spécificités techniques avantageuses (instantanéité de la mesure permettant de faire des mesures à haute fréquence, neutralité électromagnétique), les capteurs à fibre optique se placent au premier rang des technologies de monitoring pour le suivi des structures. Ils représentent à eux seul $60 \%$ du marché du structural bealth monitoring (SHM).

\section{Nombreux métiers concerinés}

L'ensemble des acteurs du BTP, de l'ingénierie et du monde de l'assurance sont concernés par les problématiques liées à l'optimisation de la maintenance et par la prévention des risques des bâtiments.

Pour les architectes, les données recueillies représentent une source de renseignements fiables pouvant être décisifs dans les étapes de construction pourvalider le comportement de matériaux, tester des méthodes innovantes ou encore préserver le patrimoine ancien. De même les gestionnaires de biens utilisent ces données pour développer des offres pour la gestion de contentieux, l'accompagnement personnalisé de clients propriétaires et gestionnaires d'infrastructures.

\section{Conclusion}

Le monde de la construction fait sien les grands défis contemporains. Construire et gérer les ouvrages aujourd'hui, c'est privilégier la protection du bâti existant, en assurer une exploitation efficiente et responsable, tout en conciliant les enjeux de la densification et ceux du développement durable. Pour relever ce défi de la santé des constructions, les technologies à base de fibre optique sont particulièrement bien placées, en complémentarité avec le potentiel du big data.

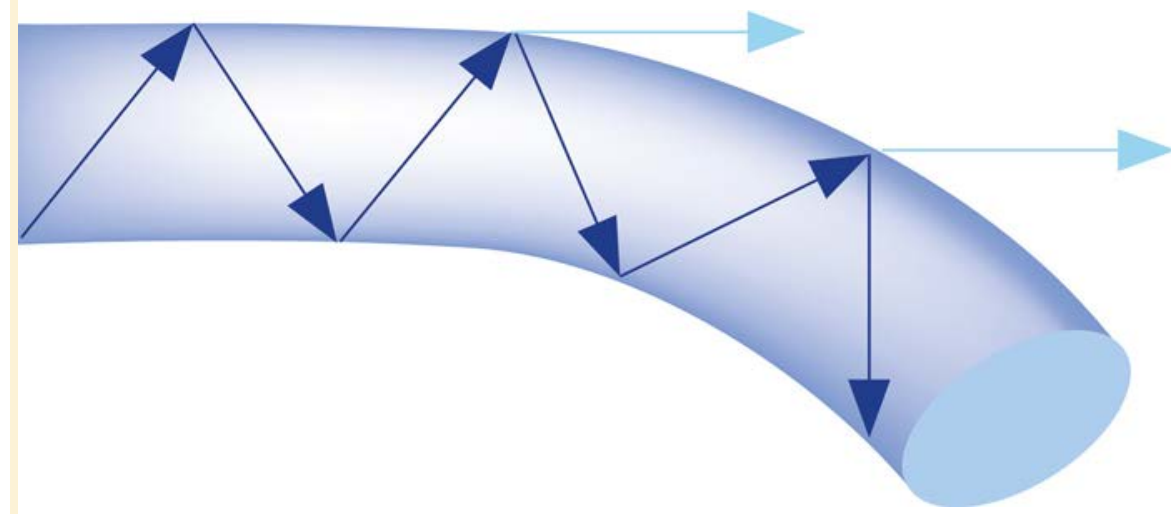

Figure 2. Parcours du flux lumineux dans la corde optique lors d'une déformation.
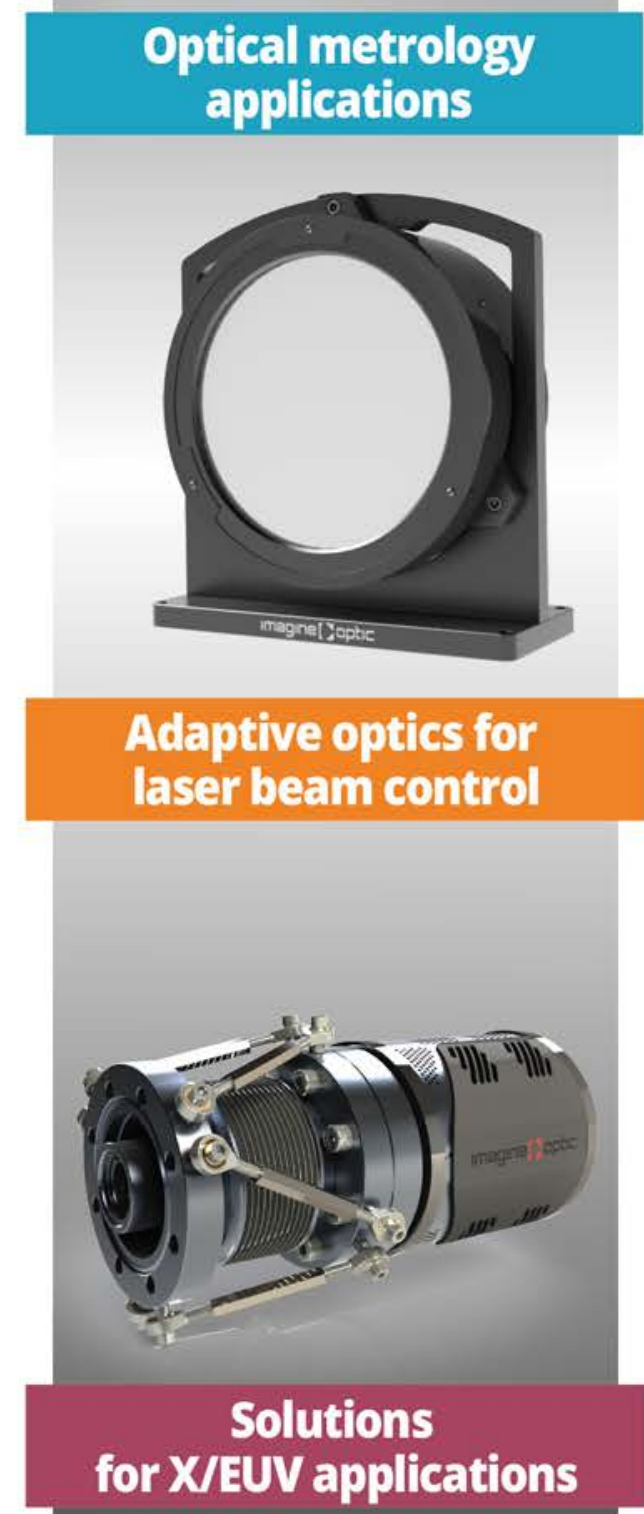

Contact us for more details: contact@imagine-optic.com or +33164861560 